\title{
Intratendinous Power Doppler Changes Related to Patient Position in Seronegative Spondyloarthritis
}

MARWIN GUTIERREZ, MD; EMILIO FILIPPUCCI, MD; WALTER GRASSI, MD, Clinica Rheumatologica, Università Politecnica delle Marche, Jesi, Ancona, Italy; MARCOS ROSEMFFET, MD, Sección de Reumatología, Instituto Municipal de Reabilitación Psicofísica, Buenos Aires, Argentina. Address correspondence to Dr. Gutierrez; E-mail: dr.gmarwin@gmail.com (J Rheumatol 2010;1057-9; doi:10.3899/jrheum.090900)

Seronegative spondyloarthropathy (SpA) is a chronic inflammatory disease that includes a heterogeneous group of diseases such as psoriatic arthritis (PsA), ankylosing spondylitis (AS), reactive arthritis, arthritis associated with inflammatory bowel disease, and undifferentiated SpA. Enthesis and tendon involvement are 2 important aspects of $\mathrm{SpA}^{1}$.

There is strong evidence that demonstrates the role of ultrasound (US) with power Doppler (PD) technique and its higher sensitivity, compared to the clinical examination, in the detection of signs of entheseal involvement in both late and early stages ${ }^{2}$. One of its main limits is operator dependency. A minimal change in the transducer position or an erroneous setting can provide completely different information, easily leading to misinterpretations.

The importance of the patient's position during the US assessment has not been well studied ${ }^{3,4}$. We investigated the effect of this phenomenon at different enthesis levels in 4 patients with SpA. US images were obtained with a MyLab 70 XVG US system (Esaote Biomedica, Genoa, Italy) with a 6-18 MHz linear transducer B-mode frequency of $18 \mathrm{MHz}$ and a Doppler frequency of 9.1 MHz. To our knowledge no similar studies have been conducted.

The first case was a 29-year-old man with entheropathic-related SpA. He reported 2 weeks of anterior knee pain. Figure 1 shows the US findings at the quadriceps tendon insertion, taken with the patient in neutral position and $30^{\circ}$ of knee flexion. The main change is characterized by complete disappearance of the intratendinous PD signal.

The second case was a 32-year-old man with PsA who had knee pain for 1 month. US examination performed at the proximal insertion of the patellar tendon, adopting both positions (neutral and flexed), showed a marked decrease of PD signal after the knee flexion (Figure 2).

The third case was a 16-year-old girl with a diagnosis of undifferentiated $\mathrm{SpA}$ and 3 months of knee pain. A dramatic change in terms of PD signal was seen when the patient adopted the knee flexion position (Figure 3).

The fourth case was a 36-year-old man with a diagnosis of AS. US examination performed at the insertion of the Achilles tendon in neutral position showed an enthesopathy associated with intense PD signal within both the tendon and the retrocalcaneal bursa. This PD finding disappeared completely after foot dorsiflexion (Figure 4).

Guidelines for the use of grayscale US in the musculoskeletal system have been developed by the European League Against Rheumatism work group ${ }^{5}$. However, no guidelines have been formulated for Doppler assessment, which appears particularly sensitive to several factors (such as setting, artifacts, and transducer pressure $)^{6}$. In specific cases, the flexion of both knee and ankle could determine an increase of the intratendinous tension, which facilitates the collapse of the microvessels. Since PD visualization at the entheseal level is an important indicator of disease activity in SpA, we suggest further consideration be given to patient position during the US examination in order to determine accurately the entheseal inflammatory involvement.

Our report provides visual evidence that patient position plays an important role in the PD assessment of entheses. Further studies of validity are required to confirm our findings.

\section{REFERENCES}

1. McGonagle D, Khan MA, Marzo-Ortega H, O’Connor P, Gibbon W, Emery P. Enthesitis in spondyloarthropathy. Curr Opin Rheumatol 1999;11:244-50.

2. D'Agostino MA, Said-Nahal R, Hacquard-Bouder C, Brasseur JL, Dougados M, Breban M. Assessment of peripheral enthesitis in the spondylarthropathies by ultrasonography combined with power Doppler: a cross-sectional study. Arthritis Rheum 2003;48:523-33.

3. Lee V, Zayat A, Wakefield RJ. The effect of joint position on Doppler flow in finger synovitis. Ann Rheum Dis 2009;68:603-4.

4. Koening MJ, Torp-Pedersen ST, Christensen R, Boesen MI, Terslev L, Hartkopp A, et al. Effect of knee position on ultrasound Doppler findings in patients with patellar tendon hyperaemia (jumper's knee). Ultraschall Med 2007;28:479-83.

5. Backhaus M, Burmester GR, Gerber T, Grassi W, Machold KP, Swen WA, et al. Working Group for musculoskeletal ultrasound in the EULAR Standing Committee on International Clinical Studies including Therapeutic Trials. Guidelines for musculoskeletal ultrasound in rheumatology. Ann Rheum Dis 2001;60:641-99.

6. Torp-Pedersen ST, Terslev L. Settings and artifacts relevant in colour/power Doppler ultrasound in rheumatology. Ann Rheum Dis 2008;67:143-9. 


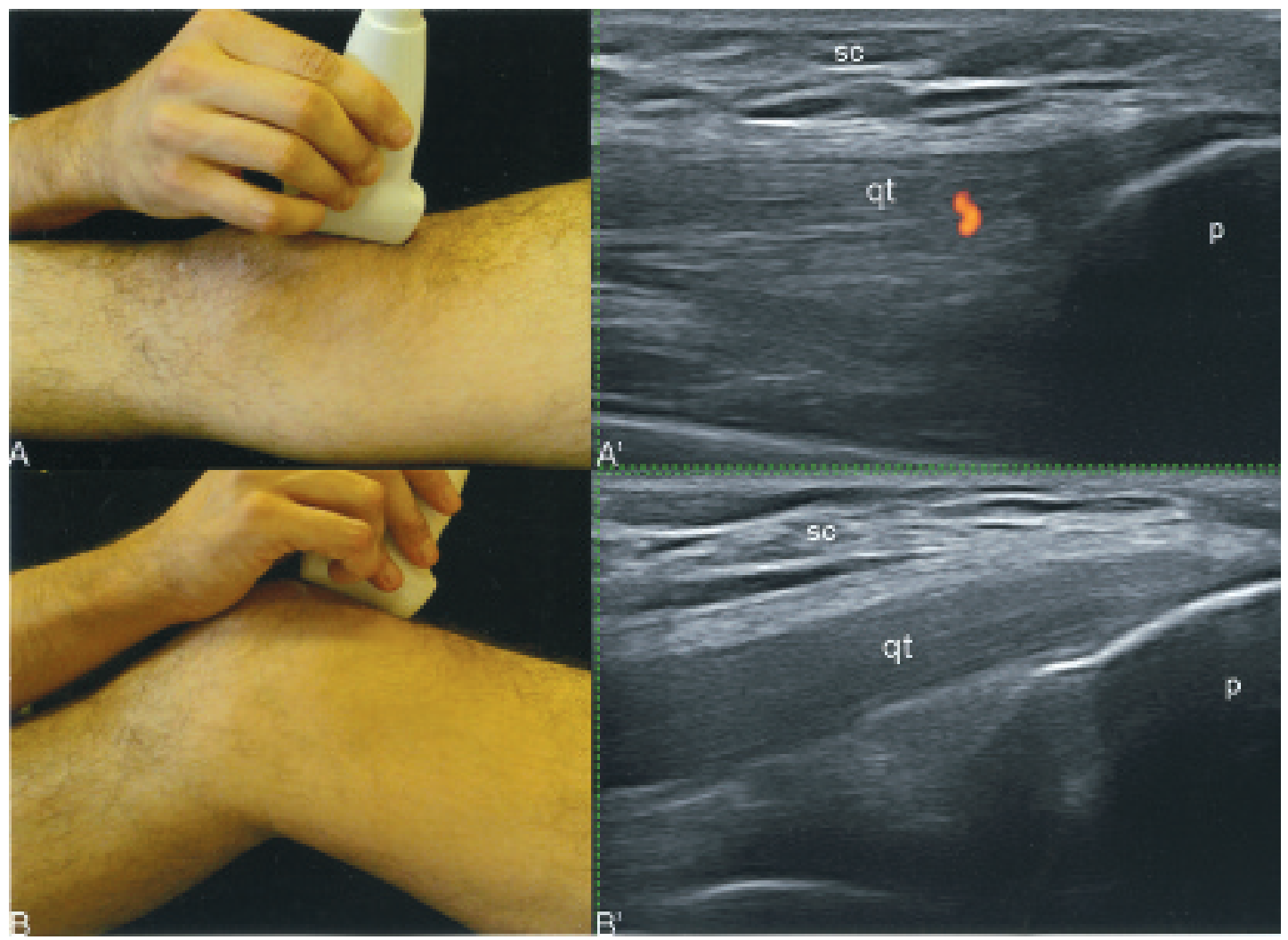

Figure 1. Entheropathic-related SpA of the knee. Insertion of quadriceps tendon into the upper pole of the patella. Photographs show the patient's position during the US examination and where the transducer was placed. A'. US examination with the patient in neutral position shows a minimal pathological PD signal within the quadriceps tendon. B'. US examination performed with $30^{\circ}$ of knee flexion. Note the complete disappearance of the PD signal. qt: quadriceps tendon; p: upper pole of patella; sc: subcutaneous tissue.

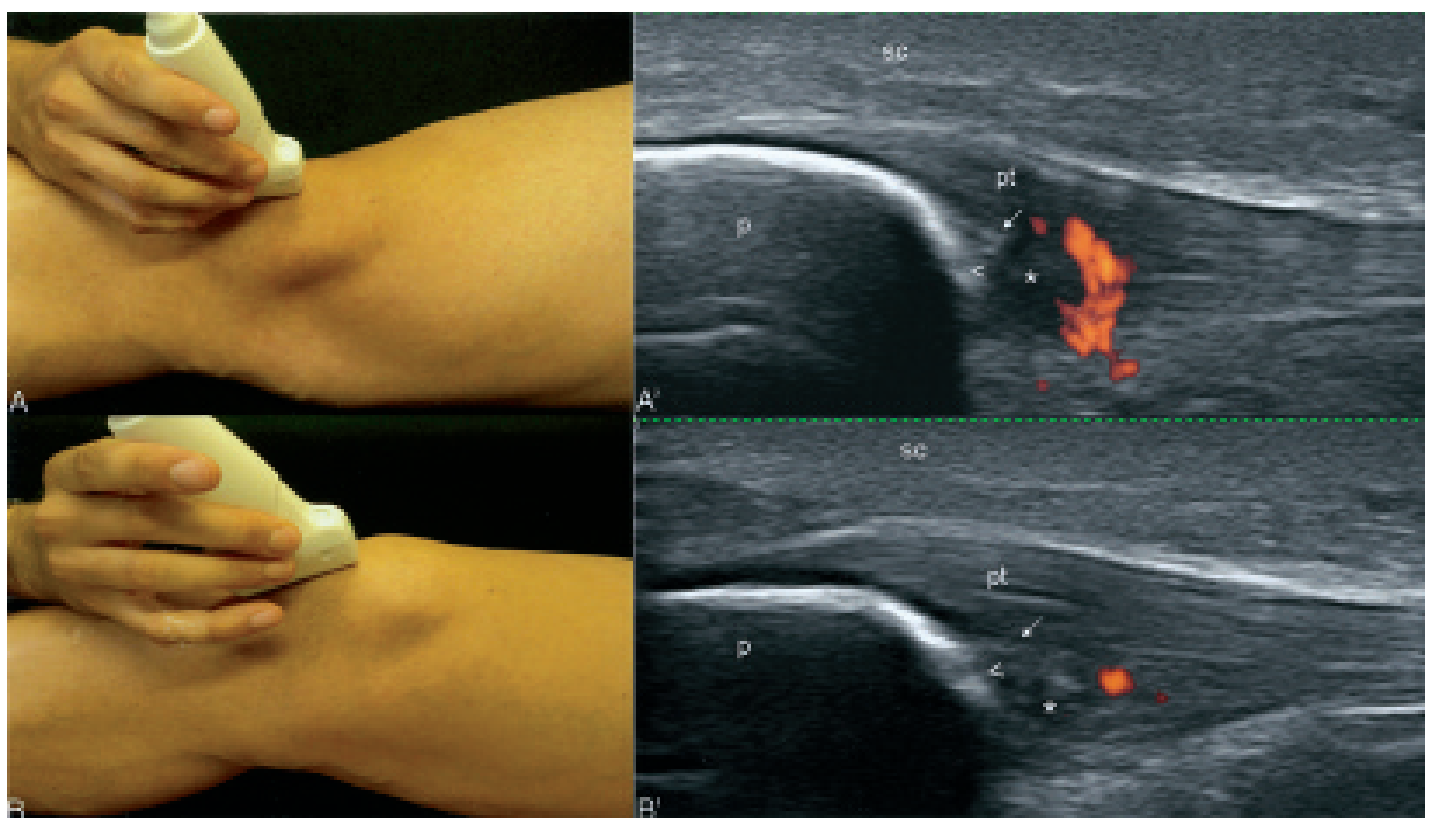

Figure 2. Psoriatic arthritis of the knee. Patellar tendon insertion into the lower pole of the patella. Photographs show the patient's position and where the transducer was placed. US examination with the patient in neutral position (A') shows a wide range of abnormalities characterized by loss of homogeneity of the echotexture tendon $(*)$, small enthesophyte (arrow), bone erosion (arrowhead), and the presence of PD signal. Subsequent US examination with knee flexed shows a dramatic reduction of the PD signal. In this position the hypoechogenicity due to anisotropy disappeared (B'), but the loss of homogeneity of the echotexture tendon $(*)$, enthesophyte (arrow), and erosion (arrowhead) persisted. p: lower pole of patella; pt: patellar tendon; sc: subcutaneous tissue.

\section{Personal non-commercial use only. The Journal of Rheumatology Copyright @ 2010 . All rights reserved.}




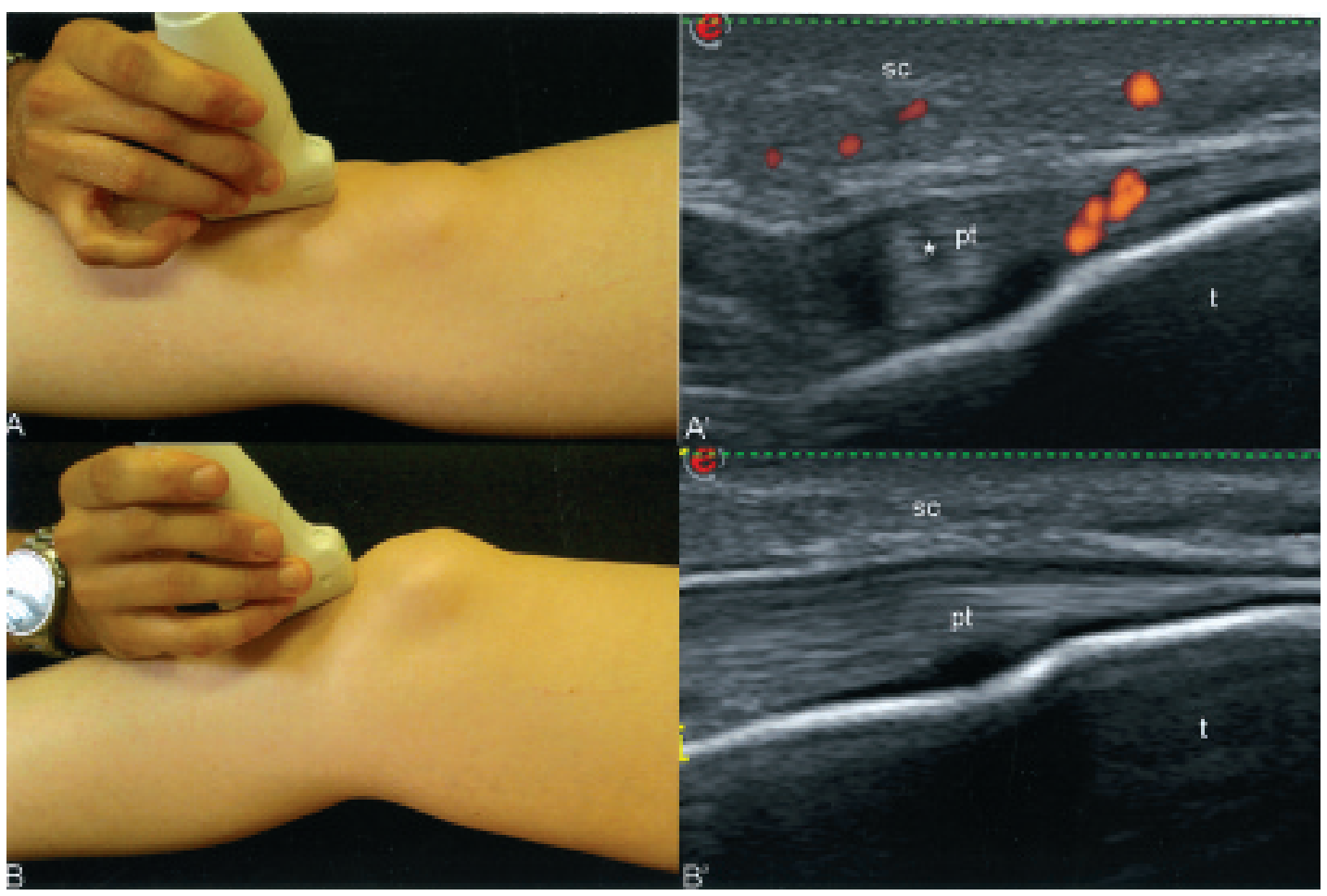

Figure 3. Undifferentiated seronegative SpA of the knee. Patellar tendon insertion into the anterior tibial tuberosity. Photographs show patient's position and location of the transducer. US examination in neutral position (A') and after $30^{\circ}$ of knee flexion (B'). A'. US examination shows a pathological PD signal within the insertional tract of the tendon, indicating active enthesitis. Note the PD signals in the soft tissues surrounding the tendon. B'. US examination performed immediately afterward shows complete disappearance of the PD signal, mimicking a normal US aspect of the tendon. t: tibial tuberosity; pt: patellar tendon; sc: subcutaneous tissue.

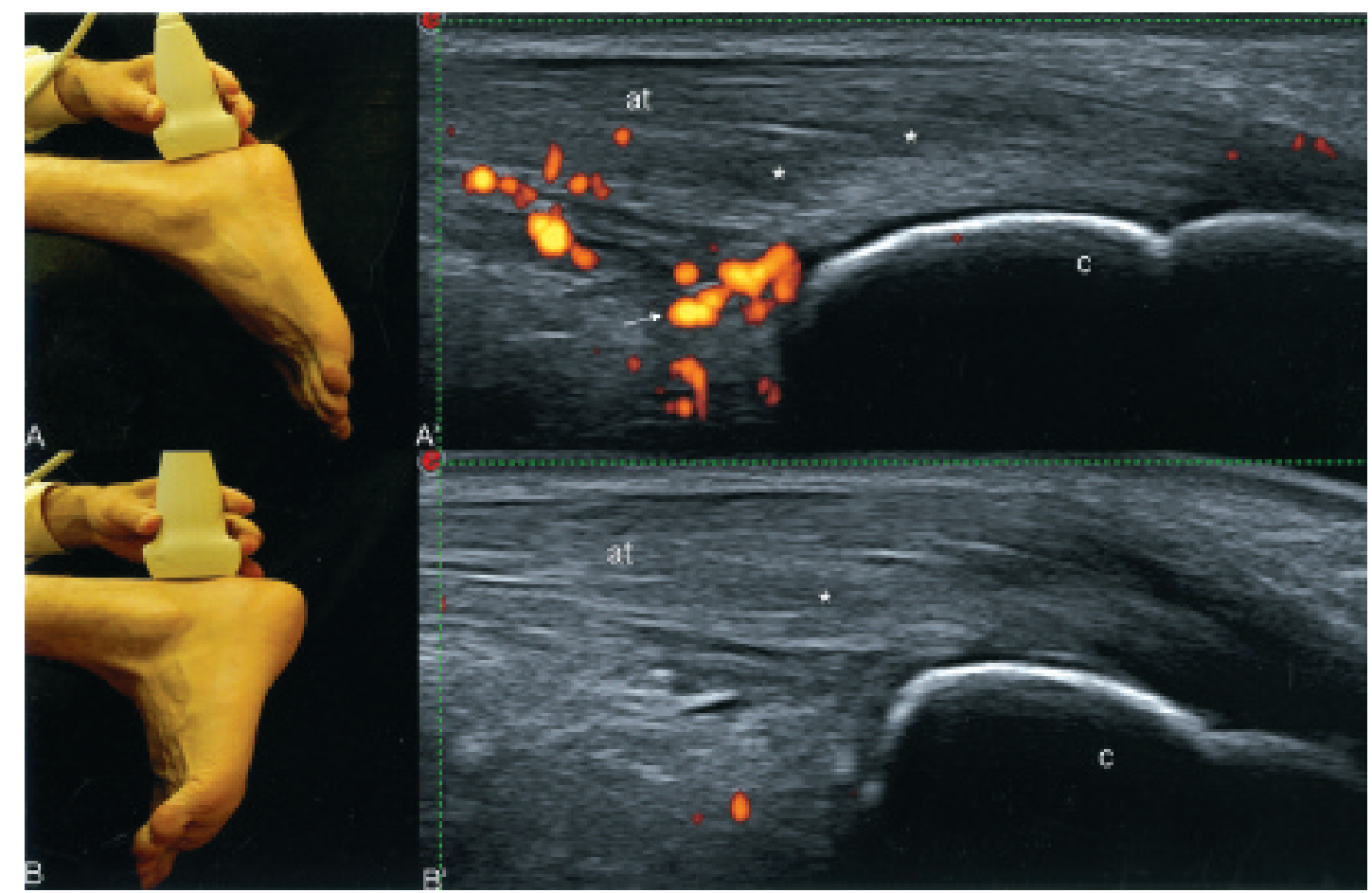

Figure 4. AS in the ankle. Achilles tendon insertion into the calcaneus bone. Photographs (A, B) illustrate patient's position and the exact point where the transducer was placed. The main grayscale US pathological findings in the neutral position (A') are represented by loss of the typical fibrillar echotexture of the tendon (*) and a small retrocalcaneal bursitis (arrow). Note the intense PD signal within both the body of the tendon and the bursa. US performed after foot dorsiflexion (B') shows complete disappearance of the intratendinous PD signal and the collapse of the retrocalcaneal bursa. c: calcaneus bone.

\section{Personal non-commercial use only. The Journal of Rheumatology Copyright @ 2010 . All rights reserved.}

